\title{
Open
}

\section{Useful pharmacodynamic endpoints in children: selection, measurement, and next steps}

\author{
Lauren E Kelly ${ }^{1}$, Yashwant Sinha ${ }^{2}$, Charlotte I S Barker ${ }^{3}$, Joseph F Standing ${ }^{3}$ and Martin Offringa ${ }^{4}$
}

Pharmacodynamic (PD) endpoints are essential for establishing the benefit-to-risk ratio for therapeutic interventions in children and neonates. This article discusses the selection of an appropriate measure of response, the PD endpoint, which is a critical methodological step in designing pediatric efficacy and safety studies. We provide an overview of existing guidance on the choice of PD endpoints in pediatric clinical research. We identified several considerations relevant to the selection and measurement of PD endpoints in pediatric clinical trials, including the use of biomarkers, modeling, compliance, scoring systems, and validated measurement tools. To be useful, PD endpoints in children need to be clinically relevant, responsive to both treatment and/or disease progression, reproducible, and reliable. In most pediatric disease areas, this requires significant validation efforts. We propose a minimal set of criteria for useful PD endpoint selection and measurement. We conclude that, given the current heterogeneity of pediatric PD endpoint definitions and measurements, both across and within defined disease areas, there is an acute need for internationally agreed, validated, and condition-specific pediatric PD endpoints that consider the needs of all stakeholders, including healthcare providers, policy makers, patients, and families.

E xpanding the evidence base for rational drug use in children presents numerous challenges, including a paucity of high-quality research on commonly used medications, complexities in designing and conducting clinical trials (CTs), and a lack of robust data on how developmental changes and disease progression affect drug exposure and response (1). Although pediatricians endeavor to deliver evidence-based pharmacotherapy, high-quality pharmacokinetic (PK) and pharmacodynamic (PD) data to inform patient management are frequently lacking. Without appropriate data on relationships between drug exposure and drug response in children and neonates, healthcare practitioners have insufficient information to definitively maximize the therapeutic benefit when prescribing drugs, while minimizing the toxicity (2).

\section{PEDIATRIC PHARMACODYNAMIC MEASURES: WHAT ARE THEY?}

PD is broadly defined as "what the drug does to the body" and is often characterized as drug response. In contrast, PK is "what the body does to the drug". PD endpoints measure a drug's activity in the body using biomarkers and/or clinical outcomes to quantify efficacy and safety (3). Although various measures of drug response are used by clinicians every day to guide therapy, PD endpoints in CTs are most often parameterized (e.g., turned into a score) so that the treatment effects can be quantitatively compared across studies. For example, a clinical question relating to mild, moderate, and severe symptoms may be transformed to a numeric rating scale from 1 to 10. This concept is discussed further in the section on Outcome-Scoring Systems.

The relationship between drug exposure and PD endpoints has been inadequately studied in children $(4,5)$. Importantly, developmental (ontogenetic) changes can affect how a drug is absorbed, distributed, and cleared from the body. This developmentally dependent variability in drug exposure affects both the desired pharmacological response and the risk of adverse effects $(6,7)$. As "pediatric" patients represent extremely diverse populations with body weights ranging from below $500 \mathrm{~g}$ to well over $70 \mathrm{~kg}$, the reporting of pharmacological research is often limited by the lack of adequate stratifications according to age or developmental stage in CT design and analysis plans (6).

A clinical example of the pediatric PD endpoint knowledge gap is in evaluating the management of an oxygen-dependent newborn diagnosed with severe pulmonary hypertension $(\mathrm{PH})$. For a pediatric study, the industry-standard, primary

\footnotetext{
${ }^{1}$ Department of Pediatrics and Child Health, Max Rady College of Medicine, University of Manitoba, Winnipeg, Manitoba, Canada; ${ }^{2}$ Therapeutic Goods Administration, Department of Health, Sydney, Australia; ${ }^{3}$ Infection, Inflammation and Rheumatology Section, UCL Great Ormond Street Institute of Child Health, University College London, London, UK; ${ }^{4}$ Child Health Evaluative Sciences, Research Institute, The Hospital for Sick Children, University of Toronto, Toronto, Ontario, Canada. Correspondence: Lauren E. Kelly (lauren.elyse.kelly@gmail.com)

L.E.K. and Y.S. conceptualized this review, designed the study, reviewed the literature and regulatory guidance, drafted the initial manuscript, and revised the final manuscript. C.I.S.B. and J.F.S. conceptualized this review, designed the study, reviewed the PD extrapolation and modeling literature, drafted the initial manuscript, and revised the final manuscript. M.O. conceptualized this review, supervised the review, designed the study, drafted the initial manuscript, and revised the final manuscript. All authors approved the final manuscript as submitted and agree to be accountable for all aspects of the work.

Received 22 December 2017; accepted 8 February 2018; advance online publication 18 April 2018. doi:10.1038/pr.2018.38
} 


\section{Review Articles | Kelly et al.}

PD endpoints for $\mathrm{PH}$ trials include exercise capacity evaluated by the 6-min walk distance (8). This PD endpoint is clearly inappropriate for newborns and pre-ambulatory children. Pediatric PH PD endpoints need to account for the age and neurodevelopmental status of the child, and -in chronic conditions-take into account how these endpoints are expected to change over time as the disease progresses (9). Previously, pediatric PH studies have used various nonstandardized primary endpoints such as cardiac catheterization parameters and bicycle-ergometry evaluation of exercise tolerance (10). With variable PD endpoints used across studies, research cannot be compared or combined to determine which treatments are most effective in the pediatric $\mathrm{PH}$ population. Identifying validated, age-appropriate, condition-specific pediatric efficacy, and safety PD endpoints is a serious challenge currently faced by pediatricians, scientists, and drug developers alike.

This paper provides an overview of PD endpoints in children while exploring the scientific principles and challenges underlying the selection and validation of robust PD endpoints in children and neonates.

\section{METHODS}

Through the StaR Child Health network (2), an expert group was convened, including pediatric pharmacology and CT experts from the United Kingdom, Australia, and Canada. The StaR Child Health network is an international consortium working to improve global standards for child health research (2). A literature review guided by an experienced research librarian was performed to identify evidence about pediatric PD endpoint selection for clinical research. The evidence was summarized to provide the basis for recommendations for selecting pediatric PD endpoints and identify current knowledge gaps. MEDLINE (Ovid) and EMBASE (Ovid) were searched from 1946 to September 2017 to identify citations potentially relevant to the topic of "standards for assessing PD endpoints in children". A search strategy incorporating the subjects of $\mathrm{PD}$, practice guidelines, and "clinical trials as topic" with age-specific limits was developed (Supplement, online only). Guidelines from regulatory authorities (European Medicines Agency (EMA), FDA, and Therapeutic Goods Administration) were reviewed for existing standards relevant to establishing and measuring PD endpoints in children. Electronic searching was limited by a lack of standardized terminology and indexing of papers in the field. The broader electronic search identified papers specific to therapeutic areas, drug classes, and individual drugs, with no general standards for PD endpoints identified (Supplementary Table 1, online).

\section{CURRENT REGULATORY GUIDANCE}

To increase the efficiency of pediatric studies and maximize the use of existing information, regulatory authorities have accepted extrapolated data from trials in other populations (mainly adults). The FDA (US Food and Drug Administration) produced draft guidance in 2014 regarding general considerations for pediatric studies for drugs and biological products (11). It includes a pediatric study planning and extrapolation algorithm relevant to PD endpoints in children, reflecting an update of the FDA pediatric study decision tree previously published in 2003 (11). The decision tree addresses the circumstances under which full or partial extrapolation can be considered in children, given a similar disease progression and response to intervention. It is noteworthy that an FDA report highlighted that only $6 \%$ of drugs reviewed could be completely extrapolated from efficacy data in adults (12). If the efficacy or toxicity endpoint is delayed, rare, or cannot be directly measured, the FDA recommends the selection of a biomarker for this purpose (11). Available data on validated pediatric biomarkers are currently limited and, again, when extrapolating biomarker endpoints from other populations, age-dependent changes in the context of pediatric disease progression require consideration (7). The FDA has also started a Letter of Support Initiative where comments from the Center for Drug Evaluation and Research on the potential value of a biomarker can be appended (13). These letters are meant to increase transparency and provide contact information for other academic, industry, or government groups to provide collaborative data.

The FDA has published a table of biomarkers used as outcomes in the evaluation of FDA-approved therapeutics available online (14). Although this is undoubtedly helpful, little information is given on the context of use, and if pediatric patients were included in the evaluated studies. The Framework for Defining Evidentiary Criteria for Biomarker Qualification drafted by the NIH Biomarkers Consortium to harmonize the biomarker qualification process is available online (15), and further details regarding the qualification process are agency specific and outside the scope of this review.

The EMA Committee for Medicinal Products for Human Use (CHMP) has published several guidelines relating to drug development and PK in children (16) and in newborns (17). These guidelines are updated periodically, drafted by working parties, and subject to public consultation from relevant stakeholders. Disease-specific guidelines sometimes contain relevant PD standards in pediatric addenda (e.g., guidelines for pulmonary arterial hypertension (18), acute cardiac failure (19), and lipid disorders (20)). These addenda acknowledge the differences in the pathophysiology of these conditions between adult and pediatric patients and provide guidance to the pharmaceutical industry regarding trial design and appropriate endpoints in pediatric studies.

Regulatory-academic-industry partnerships have formed to accelerate the development of safe and effective medicines for children. An example of this partnership is the International Neonatal Consortium (INC) that includes nurses, clinicians, researchers, industry, regulators, and parent representatives. The goal of INC is to unite stakeholders, forge a predictable regulatory path, and to develop practical tools (21) that can be incorporated into clinical trials to increase efficiency (https:// c-path.org/programs/inc/). The applicability of recommendations generated from such groups of international experts will likely extend beyond the neonatal period. Recently, INC released a white paper (22) to support investigators evaluating medicines in neonates that recommends linking PD data to PK where possible to determine the exposure-response relationships and interpreting this response in the context of available evidence in other populations. In October 2017, 


\section{Pediatric pharmacodynamic endpoints Review Articles}

the FDA awarded a grant to the Institute for the Advancement of CTs for Children (I-ACT) and the Pediatric Trials Network (PTN) based out of Duke University in the United States (23). I-ACT and PTN share a goal of providing expert advice on CT design and conduct for drugs and medical devices in children. The Global Research in Pediatrics (GRiP) Network of Excellence, funded by the European Union has developed guidance and research tools for pediatric studies (24-28) that can be found in their publication repository (http://www.grip-network.org/index.php/cms/en/publications). The advantages of these international partnerships include harmonization, development of best research practice, and a global union of expertise and experience in pediatric clinical research.

\section{SELECTING AND MEASURING USEFUL PD ENDPOINTS}

Useful PD endpoints are defined as measures, which, when collected systematically, can inform decision making at the bedside, by policy makers and by health authorities (regulators). Several identified considerations that are relevant to selecting and measuring useful PD endpoints in children, infants, and neonates that include the use of biomarkers, modeling and extrapolation, compliance, outcome-scoring systems, and measurement validation are summarized below.

\section{Biomarkers as PD Endpoints}

A biomarker is defined as a "characteristic that is objectively measured and evaluated as an indicator of normal biologic processes, pathogenic processes, or pharmacologic responses to a therapeutic intervention" $(29,30)$. Systematic reviews have identified a wide variety of biomarkers that have been used to measure response (31-33). Historically, many biomarkers validated for use in adults were inappropriately extrapolated to children without careful assessment of the influence of the developmental aspects of pathogenesis, ontogeny, and therapeutic response (7). There remains a dearth of validated biomarkers in pediatrics and neonatology, which has contributed to the "PD knowledge gap" recognized in pediatric drug development $(34,35)$. Validated biomarkers facilitate prediction of disease progression and treatment response. Importantly, they can provide useful surrogate endpoints within the trial design, when they have reliably demonstrated that they can predict the relevant clinical outcomes (35). In order for biomarkers to serve as valid pediatric PD endpoints, changes in biomarker concentrations must also predict beneficial or harmful clinical response in the population of interest (22).

The use of biomarkers in clinical trials is highly context specific, and regulators require that high standards of validation be met to accept biomarker data during the marketing authorization process. For example, the FDA (36) and EMA $(37,38)$ launched formal biomarker qualification processes and provide scientific advice to support innovation in this field. In an effort to disseminate information regarding the established biomarkers, the FDA established the BEST (Biomarkers, EndpointS, and other Tools) (36) resource that contains examples and explanations. During the FDA's review of new molecular entities, several studies reported the use of biomarkers as outcomes in clinical trials (39). For example, biomarkers used to evaluate inborn errors of metabolism included blood cell count, growth, serum low-density lipoprotein-C, blood phenylalanine, forced vital capacity, hemoglobin, plasma concentrations of ammonia, glutamine, and citrulline, as well as splenic volume measured by MRI (39).

In situations where a biomarker is known to change with age as a child develops, an a priori correction may be applied to account for age. One such method is to convert biomarker measures into a $z$-score. A $z$-score follows the standard normal distribution (i.e., mean of 0 and standard deviation of 1$)$. Hence, the $z$-score gives the magnitude and direction of deviation from the expected value for age in standarddeviation units, with a $z$-score of zero representing a child having the expected biomarker value for their age. An example of $z$-scoring in PD modeling is the measurement of CD4 T-lymphocyte count in HIV-infected patients treated with antiretrovirals. The adaptive immune system develops rapidly during early childhood, and while the thymus may cease growing after 1 year, changes in the epithelial space occur over the first two decades of life. The implication of this is that the normal CD4 T-cell count drops threefold during childhood, stabilizing in early adolescence (40). CD4 T-cell reconstitution following antiretroviral initiation has been modeled using $z$-scores (41). One potential criticism of $z$ score conversion is the strong underlying assumption that the study population has the same age-dependent distribution values as an available reference population from which the $z$ score is calculated (42). Where appropriate, juvenile animal models may shed light on the relationship between growth and development with variability in PD endpoints.

When evaluating biomarkers in pediatric drug development, clinical, regulatory, and methodology experts should be consulted as early as possible in the study-design process. Biomarkers must be developed and tested within a specific context of use in drug development that includes the class of biomarkers and the specific research question (33). The financial cost of validating biomarkers to meet regulatory standards and the time frames involved must not be underestimated. This is an area where dedicated funding streams will be critical to enable sustained progress in biomarker development and to underpin future progress in pediatric therapeutics.

\section{Modeling: Extrapolating Exposure-Response Relationships}

Several challenges arise when extrapolating exposureresponse (PK-PD) relationships across indications and agegroups. One fundamental source of uncertainty is a lack of reliable data on disease progression, particularly in neonates and young children who were historically excluded from clinical research. Without clear knowledge of the differences in disease progression, it is problematic to use one population to predict clinical response in another (43). Although it is 


\section{Review Articles | Kelly et al.}

increasingly possible to make assumptions about the impact of age-related changes in enzymes, transporters, and organ function on a drug's PK, it is often less clear how these variables affect PD response (44). A further challenge occurs when several medications with multiple mechanisms of action are coadministered, such as combined chemotherapy in oncology.

Modeling and simulation are increasingly required by regulators to inform the design and conduct of $\mathrm{PK} / \mathrm{PD}$ studies for drugs used in pediatric populations $(11,45)$. A recent report by Mulugeta et al., identified 31 products approved in children by the FDA between 1998 and 2012 with full (39\%) or partial (61\%) extrapolation (43). Most of these products $(n=25 / 31)$ were studied in more than one other pediatric age group (37). PD can be modeled alone, or simultaneously with PK $(25,46,47)$. A detailed overview of the principles of PKPD modeling is available elsewhere (47-50). In brief, a PK model describes the relationship between the drug-dosing regimen and the plasma drug concentration time profile, whereas a PD model describes the relationship between drug concentration and the biological effect (48). Mathematical techniques for creating models of the interactions between developmental physiology, PK, PD, and disease (pharmacometrics) have become increasingly sophisticated in recent years, resulting in the successful application of $\mathrm{PD}$ models in pediatric drug development and post-marketing research (47,51-54).

Furthermore, PKPD models can be used to inform the design of comparative randomized control trials $(45,53,55)$. In some cases, simulation and extrapolation from robust adult PKPD data are sufficient for licensing medicines in children, for example, esomeprazole for gastroesophageal reflux disease in children aged 1-17 years (56). PKPD models can play an important role in determining if the expected outcome effect size will be similar in children of different ages and make the best use of available data in other populations (57). For example, the new class of antidiabetic medications sodiumglucose cotransporter 2 (SGLT2) inhibitors exert their effects by promoting renal glucose elimination, through reduction of glucose reabsorption in the proximal tubule (58). Adolescent patients with type- 2 diabetes tend to have superior renal function to adult patients, and hence SGLT2 inhibitors will potentially be more effective at equivalent doses. Quantifying this with modeling can thereby reduce CT sample size requirements using a predicted increased effect size in the adolescent age-group.

\section{Compliance and PD Response}

Patient adherence with the therapeutic intervention can influence the evaluation of treatment differences between study arms. Sophisticated research into pediatric PD in explanatory trials needs to be accompanied by consideration of medication adherence, particularly in ambulatory settings and in complex diseases. Although adherence is often considered separately from PD endpoints of treatment success, the consequences of disregarding patient adherence (or compliance) during the early phases of drug development are wide-reaching as nonadherence to treatment regimens can reduce or prevent the detection of treatment efficacy, thus having an impact (potentially significantly) upon trial results (59). Achieving medication adherence can be highly challenging in the pediatric setting (60). Various innovative ways of evaluating adherence in pediatric clinical studies, including electronic measurement (61), and measurements of drug and metabolites through various media $(62,63)$ merit further research.

\section{Outcome-Scoring Systems}

Scoring systems, which assign numerical merit to outcomes to estimate the degree of a clinical situation, remain popular in pediatric medicine $(53,64)$. Scoring systems can support pediatric PD measurement in CTs by combining several isolated measures into one score to monitor disease status before and after therapeutic intervention(s). However, scoring systems derived for adults again may not be suitable or valid for use in the pediatric population (65). Dedicated age-specific scoring systems need to be developed and then validated for use as endpoints in CT settings, as well as within clinical practice (65-67). Examples of age-appropriate PD-related scoring systems include the COMFORT-B score (68) used to measure sedation in pediatric critical care and the Premature Infant Pain Profile (69) used to measure pain in nonverbal children. In adults, the gold standard for measuring pain is self-report, which is clearly inappropriate for younger age groups. The COMFORT-B score is made up of six items: alertness, calmness, respiratory response (for ventilated children) or crying (for spontaneously breathing children), body movements, facial tension, and muscle tone. Each item is rated on a scale $1-5$ so that the total score ranges from 6 to 30 . The COMFORT-B score has been validated in this clinical setting, and if CT use of COMFORT-B is planned, outcome assessors should be trained with video assessment and test patients (68). Satisfactory results compared with an experienced scorer to measure inter-rater reliability $(70,71)$ should be achieved before collecting PD CT data. However, while scoring systems confer the advantage of combining multivariate data into a univariate quantity (72), they sacrifice the granularity of detail within the data. Ideally, it is preferable to retain all raw data used to generate composite scores whenever feasible, to facilitate more detailed statistical analysis. For example, the Finnegan Score (73) for neonatal abstinence syndrome includes 21 signs and symptoms. If raw data are maintained, investigators may be able to answer more specific research questions regarding the intervention's effect on generalized convulsions (one of the score items).

\section{Validation of PD Endpoints}

Although the validation process presents practical and financial challenges, it is essential to ensure that PD endpoints are meaningful, reproducible, and relevant (3). There are several examples of validated pediatric PD endpoints (5). The aforementioned COMFORT-B score presents a useful 


\section{Pediatric pharmacodynamic endpoints Review Articles}

example. Given the potential limitations of a scoring system vulnerable to interobserver variability, Ista et al. (74) ensured reliability by training PICU nurses with video material and bedside instructions. Nurses new to the scoring system underwent repeated assessments with a trained nurse to maximize the fidelity of their scoring. Such a rigorous approach to staff training benefits the application of scoring system-based PD endpoints in both clinical and research settings, and is strongly recommended for all PD endpoints that depend on proxy (healthcare professionals' or parents') observations or measurements.

In the analytical setting, the validation of laboratory biomarker assays can present additional difficulties. This was demonstrated in the management of type- 1 diabetes, where long-term monitoring of $\mathrm{HbA}_{1 \mathrm{c}}$ (glycated hemoglobin) was affected by technical aspects of the measurement methods. This led to speculation over whether the differences in patients' longitudinal $\mathrm{HbA}_{1 \mathrm{c}}$ measurements were due to the laboratory assays rather than differences in clinical management strategies: details of the $\mathrm{HbA}_{1 \mathrm{c}}$ assay laboratory and equipment factors are discussed elsewhere (75-78). These experiences highlight the importance of the new regulatory guidelines on bioanalytical method validation $(79,80)$, as well as inter-laboratory proficiency testing and methodological standardization for both old and new biomarker assays (78). Quantitative biomarker assays should be reliable, selective, and validated under conditions equivalent to good laboratory practice whenever feasible (3). For multicenter-CTs, transference studies should be planned prospectively to quantify variability related to laboratory instrumentation.

Clinical aspects of validation include the definition of the normal ranges/values of PD markers (including biomarkers) in both healthy children and the target patient population. The latter can be difficult, given the rarity of many pediatric diseases, which affect small, heterogeneous patient populations (81). The identification of age-matched controls can be challenging. Developing suitable pediatric reference value distributions thus requires dedicated research in its own right
(82). There is ongoing research into the identification and validation of pediatric biomarkers in different subspecialist clinical contexts (32,83-85). For example, there have been rapid advances in candidate biomarkers for renal function and acute kidney injury in children, such as cystatin C, neutrophil gelatinase-associated lipocalin, and kidney injury molecule 1 (86-88), although translation from research into clinical practice has been slow (89). The use and uptake of validated biomarkers will be improved by efforts of regulators to swiftly disseminate new information and updates regarding biomarkers relevant to both clinical practice and CT outcomes in children.

\section{IDENTIFYING A USEFUL PD ENDPOINT}

PD endpoints must be able to consistently quantify the clinical response of a child to a specific intervention, as measured at a specific stage of development. Valid PD endpoints are described within the context of the "four Rs": relevant, responsive, reproducible, and reliable $(90,91)$. The characteristics of an ideal PD endpoint are summarized in Table 1. PD endpoints must be clinically relevant and, where possible, should take into account how the patient feels and functions (92). PD endpoints must be responsive to changes in clinical progression over time; this characteristic is essential to track disease improvements and treatment progression (92).

To ensure validity, PD endpoints should be reproducible, for example, in patients of different ethnicities. Due to agedependent variability in rates of disease progression and organ function in children, different PD endpoints in specific age groups (e.g., neonates, toddlers, and adolescents) are typically required. Within these age groups, $\mathrm{PD}$ endpoints must be reliable and should be similar, regardless of the environment or the person who takes the measurement. To determine which PD endpoints should be evaluated, we recommend undertaking a systematic review and using a consensus process to select outcomes and measurement tools. Patients and families, as well as policy makers should be

Table 1. Seven characteristics of useful PD endpoints

1. Meaningfully describe the patient's pharmacological and clinical responses to drug therapy with respect to

(a) Incorporating both harms and benefits

(b) Accounting for patient and families well-being (quality of life)

2. Can be interpreted against data extrapolated from other diseases or age-groups and existing scientific literature

3. Can be used to answer the research question while informing healthcare decision making at the bedside and policy level

4. Is responsive to change and comes with a defined age-specific minimally important difference

5. Is reproducible and, where possible, objective

6. Can be consistently and reliably measured by outcome assessors

7. Has an established age-appropriate validated measure with established reference ranges in the specific age group and disease state 8. Is feasible with respect to

(a) Acceptability in terms of burden on the child or caregivers with minimal compliance/adherence concerns

(b) Timing: where possible combined with routine tests

(c) Cost considerate: license, equipment, and skill set of the outcome assessor 


\section{Review Articles | Kelly et al.}

Table 2. Six disease-specific research activities needed to advance pediatric pharmacodynamics

1. Develop, test, and implement validation strategies for pharmacodynamic measurement tools (including biomarkers), especially in younger agegroups

2. Harmonize the definitions of disease and disease severity (if not already agreed)

3. Develop methods (akin to allometric scaling in pharmacokinetics) for robust scaling of pharmacodynamic endpoints with known age- or size-related factors

4. Identify the optimal pharmacodynamic study design and sampling times

5. Develop and test the study design methods for determining what outcomes are important to patients, families, and policy makers

6. Determine baseline "normal" values for biomarkers accounting for developmental status and disease progression

7. Develop, test, and implement MeSH terms for indexing of papers, developing, and validating pharmacodynamic measures in child health trials to ensure that future literature searches will identify these articles

included in the process to select meaningful PD endpoints. Methods for the development of disease-specific core outcome sets are described by Williamson et al. (93). A repository of core outcome sets is available online through the Core Outcome Measures in Effectiveness Trials (COMET) initiative (http://www.comet-initiative.org/). If a core outcome set exists for the condition of interest, it should be employed, whereas additional relevant PD endpoints may be added.

\section{CURRENT PD RESEARCH NEEDS}

Stemming from a review of the currently available regulatory and scientific evidence, recommendations for future research for specific disease areas are presented in Table 2. In order to increase the quality, consistency, and usefulness of PD endpoints, targeted methodology research is needed. For example, as outlined above, scoring systems comprising a composite of several measures have a risk of losing information since different combinations of subscores can give the same overall score. Item response theory approaches could be useful in teasing out contributions of the individual subscores to the PD response (72). The heterogeneity of definitions of $\mathrm{PD}$ endpoints exacerbates the difficulty in introducing generic recommendations; however, efforts to harmonize approaches internationally will begin to address these issues.

A further challenge for synthesizing scientific literature and guidance in this area includes a lack of appropriate and valid search terms. Ideally, the development of MeSH search terms would improve the ability of future researchers to identify, compare, and contrast pediatric PD marker validation studies. As the validation of $\mathrm{PD}$ endpoints often requires large sample sizes, there is an important role for industry-academicregulatory partnerships to moving the science behind selecting valid PD endpoints forward. Data from previous studies where PK-PD may not be the primary objective, could potentially be used by researchers to better characterize the $\mathrm{PK}-\mathrm{PD}$ relationships in pediatric diseases where such information is lacking. This will require open data-sharing agreements and partnerships. Additional research is needed into the ability of real-world evidence (routine laboratory tests, patient registries, and electronic health records) to support the selection of high-quality pragmatic pediatric PD endpoints. Recently, the use of patient-reported outcomes in clinical practice has increased; however, the validation of patient-reported outcomes to meet regulatory requirements should be explored. Finally, how to best engage patients and families in the process of selecting PD endpoints requires further evaluation.

\section{CONCLUSIONS}

The importance of utilizing appropriate pediatric PD endpoints, including biomarkers, and facilitating their validation in children is clear. Although there is increasing knowledge of $\mathrm{PK}$ in neonates and children, there is a paucity of information related to $\mathrm{PD}$. Based on the available evidence, no overall recommendation for the selection of pediatric $\mathrm{PD}$ endpoints in CTs can be provided at this stage. However, we provide criteria for selecting and measuring useful PD endpoints for each age-disease-specific group, and set an agenda for research in this field. Engaging with regulators early in the development process will help ensure that endpoints meet the regulatory validation requirements. Trial sponsors and regulators need to agree to use early-phase trials to validate candidate PD endpoints, including biomarkers, to advance the field. Across these domains, PK/PD modeling enhances both pediatric PD research and trial design, and, where resources permit, model-based approaches should underpin future pediatric $P D$ research.

\section{ACKNOWLEDGMENTS}

We thank Ms. Allison Needham, Child Health Evaluative Sciences, Research Institute, The Hospital for Sick Children, and University of Toronto, Toronto, Ontario, Canada for her assistance with the project management of this manuscript. Ms. Wei Qi, reference librarian, Information Resources and Research Services, Regulator Knowledge and Technology Services Branch, and Department of Health, Australia and Ms. Tamsin Adams-Webber, manager, Library and Archives Services, Learning Institute, and Hospital for Sick Children, Toronto, Ontario, Canada kindly provided advice for the search strategy. We thank Dr. Lee Dupuis for providing an expert review of this manuscript. We also acknowledge the Star Child Health Group, of which all authors are members. Standards for Research (StaR) in Child Health was founded in 2009 to address the paucity and limitations of pediatric clinical trials. This initiative brings together international experts who are committed to developing practical, evidence-based standards to enhance the quality, ethics, and relevance of clinical research in child health. More information can be found at the Star Child Health Website (www.starchildhealth.org). 
STATEMENT OF FINANCIAL SUPPORT:

No specific funding was used for the preparation of this manuscript. The research leading to these results has received funding from the European Union's Seventh Framework Programme for research, technological development, and demonstration under grant agreement no. 261060 (Global Research in Paediatrics-GRiP Network of Excellence), which funded C.I.S.B. as a Clinical Research Fellow. She has also received funding from a National Institute for Health Research (NIHR) Academic Clinical Fellowship (ACF-2016-18-016). L.E.K. is supported by the Children's Hospital Research Institute of Manitoba. J.F.S. has received funding from United Kingdom Medical Research Council Fellowships (grants G1002305 and M008665). C.I.S.B. and J.F.S. have been supported by the NIHR Biomedical Research Centre at Great Ormond Street Hospital for Children NHS Foundation Trust and University College London.

Disclosure: The authors declare no conflict of interest.

\section{REFERENCES}

1. Canadian Council of Academies Improving Medicines for Children Expert Panel on Therapeutic Products for Infants, Children and Youth, 2014. http://www.scienceadvice.ca/uploads/eng/assessments\%20and\% 20publications\%20and\%20news\%20releases/therapeutics/therapeutics_ fullreporten.pdf.

2. Klassen TP, Hartling L, Hamm M, van der Lee JH, Ursum J, Offringa M. StaR Child Health: an initiative for RCTs in children. Lancet 2009;374: $1310-2$.

3. Colburn WA, Lee JW. Biomarkers, validation and pharmacokineticpharmacodynamic modelling. Clin Pharmacokinet 2003;42:997-1022.

4. Holford N. Dosing in children. Clin Pharmacol Ther 2010;87:367-70.

5. Mulla $\mathrm{H}$. Understanding developmental pharmacodynamics: importance for drug development and clinical practice. Paediatr Drugs 2010;12: 223-33.

6. Williams K, Thomson D, Seto I, et al. Standard 6: age groups for pediatric trials. Pediatrics 2012;129 (Suppl 3): S153-60.

7. Goldman J, Becker ML, Jones B, Clements M, Leeder JS. Development of biomarkers to optimize pediatric patient management: what makes children different? Biomark Med 2011;5:781-94.

8. Gaine S, Simonneau G. The need to move from 6-minute walk distance to outcome trials in pulmonary arterial hypertension. Eur Respir Rev 2013;22:487-94.

9. Adatia I, Haworth SG, Wegner M, et al. Clinical trials in neonates and children: Report of the pulmonary hypertension academic research consortium pediatric advisory committee. Pulm Circ 2013;3:252-66.

10. Nicolarsen J, Ivy D. Progress in the diagnosis and management of pulmonary hypertension in children. Curr Opin Pediatr 2014;26:527-35.

11. Food and Drug Administration FDA General Pharmacology Considerations for Drugs and Biological Products. Guidance for Industry. Draft Guidance, 2014 http://www.fda.gov/downloads/drugs/ guidancecomplianceregulatoryinformation/guidances/ucm425885.pdf.

12. Dunne J, Rodriguez WJ, Murphy MD, et al. Extrapolation of adult data and other data in pediatric drug-development programs. Pediatrics 2011;128:e1242-9.

13. Food and Drug Administration Letters of Support Initiative, 2017 (cited 02 February 2018) https://www.fda.gov/Drugs/DevelopmentApprovalProcess/ ucm434382.htm.

14. Food and Drug Administration Biomarkers Used as Outcomes, 2017 (cited 28 Nov 2017) https://www.fda.gov/Drugs/DevelopmentApprovalProcess/Drug DevelopmentToolsQualificationProgram/BiomarkerQualificationProgram/ ucm535926.htm.

15. Biomarkers Consortium Evidentiary Standards Working Group Framework for Defining Evidentiary Criteria for Biomarker Qualification, 2016 (cited 28 Nov 2017] https:/fnih.org/sites/default/files/final/ pdf/Evidentiary\%20Criteria\%20Framework\%20Final\%20Version\%20Oct \%2020\%202016.pdf.

16. European Medicines Agency Committee for Medicinal Products for Human Use. Guidelines on the Role of Pharmacokinetics in the Development of Medicinal Products in the Paediatric Population, 2006
http://www.ema.europa.eu/docs/en_GB/document_library/Scientific_ guideline/2009/09/WC500003066.pdf.

17. European Medicines Agency Committee for Medicinal Products for Human Use. Guideline on the Investigation of Medicinal Products in the Term and Preterm Neonate, 2007 http://www.ema.europa.eu/docs/en_ GB/document_library/Scientific_guideline/2009/09/WC500003754.pdf.

18. European Medicines Agency Paediatric Addendum to the CHMP Guideline in the Clinical Investigations of Medicinal Products for the Treatment of Pulmonary Hypertension, 2011 http://www.ema.europa.eu/docs/en_GB/ document_library/Scientific_guideline/2012/02/WC500122492.pdf.

19. Sokalska A, Anderson M, Villanueva J, et al. Effects of simvastatin on retinoic acid system in primary human endometrial stromal cells and in a chimeric model of human endometriosis. J Clin Endocrinol Metab 2013;98:E463-71.

20. European Medicines Agency Paediatric Addendum to CHMP Guideline on Clinical Investigation of Medicinal Products in the Treatment of Lipid Disorders, 2012 http://www.ema.europa.eu/docs/en_GB/document_library/ Scientific_guideline/2012/10/WC500133180.pdf.

21. Davis JM, Turner MA. Global collaboration to develop new and existing drugs for neonates. JAMA Pediatr 2015;169:887-8.

22. Ward RM, Benjamin D, Barrett JS, et al. Safety, dosing, and pharmaceutical quality for studies that evaluate medicinal products (including biological products) in neonates. Pediatr Res 2017;81: 692-711.

23. EurekAlert US FDA Awards I-ACT for Children a Grant for Pediatric Medicines \& Devices Network, 2017 (cited 28 November 2017] https:// www.eurekalert.org/pub_releases/2017-10/zsms-ufa102417.php.

24. Kelly LE, Davies EH, Saint-Raymond A, Tomasi P, Offringa M. Important issues in the justification of a control treatment in paediatric drug trials. Arch Dis Child 2016;101:962-7.

25. Vermeulen E, Anker JN, Della Pasqua O, Hoppu K, Lee JH. How to optimise drug study design: pharmacokinetics and pharmacodynamics studies introduced to paediatricians. J Pharm Pharmacol 2017;69:439-47.

26. Smania G, Baiardi P, Ceci A, Magni P, Cella M. Model-based assessment of alternative study designs in pediatric trials. Part I: frequentist approaches. CPT Pharmacometrics Syst Pharmacol 2016;5:305-12.

27. Smania G, Baiardi P, Ceci A, Cella M, Magni P. Model-based assessment of alternative study designs in pediatric trials. Part II: Bayesian approaches. CPT Pharmacometrics Syst Pharmacol 2016;5:402-10.

28. Osokogu OU, Dodd C, Pacurariu A, Kaguelidou F, Weibel D, Sturkenboom MC. Drug safety monitoring in children: performance of signal detection algorithms and impact of age stratification. Drug Saf 2016;39:873-81.

29. Wagner JA. Biomarkers: principles, policies, and practice. Clin Pharmacol Ther 2009;86:3-7.

30. Kearns GL, Artman M. Functional biomarkers: an approach to bridge pharmacokinetics and pharmacodynamics in pediatric clinical trials. Curr Pharm Des 2015;21:5636-42.

31. Bai JP, Barrett JS, Burckart GJ, Meibohm B, Sachs HC, Yao L. Strategic biomarkers for drug development in treating rare diseases and diseases in neonates and infants. AAPS J 2013;15:447-54.

32. Kelly LE, Caswell K, Short MA, Parimi PS, Offringa M, Diacovo T. Response biomarkers in neonatal intervention studies. Pediatr Res 2017;83:425-30.

33. Amur S, Frueh FW, Lesko LJ, Huang S-M. Integration and use of biomarkers in drug development, regulation and clinical practice: a US regulatory perspective. Biomark Med 2008;2:305-11.

34. Lake F. Pediatric-specific biomarkers: an important but challenging field. Biomark Med 2012;6:237-8.

35. Kearns GL. Beyond biomarkers: an opportunity to address the 'pharmacodynamic gap' in pediatric drug development. Biomark Med 2010;4:783-6.

36. FDA-NIH Biomarker Working Group BEST (Biomarkers, EndpointS and other Tools) Resource, 2016 (cited 27 October 2017) https://www.ncbi. nlm.nih.gov/books/NBK402286.

37. European Medicines Agency Committee for Medicinal Products for Human Use. Final Conclusions of the Pilot Joint EMEA/FDA VXDS 
Experience on Qualification of Nephrotoxicity Biomarkers, 2009 http:// www.ema.europa.eu/docs/en_GB/document_library/Regulatory_and_ procedural_guideline/2009/10/WC500004205.pdf.

38. Manolis E, Koch A, Deforce D, Vamvakas S. The European Medicines Agency experience with biomarker qualification. Methods Mol Biol 2015;1243:255-72.

39. Food and Drug Administration Biomarkers Used as Outcomes, 2017 https:/www.fda.gov/Drugs/DevelopmentApprovalProcess/

DrugDevelopmentToolsQualificationProgram/

BiomarkerQualificationProgram/ucm535926.htm.

40. Bains I, Thiebaut R, Yates AJ, Callard R. Quantifying thymic export: combining models of naive $\mathrm{T}$ cell proliferation and TCR excision circle dynamics gives an explicit measure of thymic output. J Immunol 2009;183:4329-36.

41. Lewis J, Walker AS, Castro H, et al. Age and CD4 count at initiation of antiretroviral therapy in HIV-infected children: effects on long-term T-cell reconstitution. J Infect Dis 2012;205:548-56.

42. Hoare R, Callard R, Standing Jeds.Modelling CD4 T Cell Reconstitution in HIV-Infected Children Starting Antiretroviral Therapy. Population Approach Group in Europe, 2016; Croatia www.page-meeting.org/? abstract $=3425$ Accessed July 2016.

43. Mulugeta Y, Barrett JS, Nelson R, et al. Exposure matching for extrapolation of efficacy in pediatric drug development. J Clin Pharmacol 2016;56:1326-34.

44. Brouwer KL, Aleksunes L, Brandys B, et al. Human ontogeny of drug transporters: review and recommendations of the pediatric transporter working group. Clin Pharmacol Ther 2015;98:266-87.

45. Barker CI, Germovsek E, Hoare RL, Lestner JM, Lewis J, Standing JF. Pharmacokinetic/pharmacodynamic modelling approaches in paediatric infectious diseases and immunology. Adv Drug Deliv Rev 2014;73: 127-39.

46. Nielsen EI, Friberg LE. Pharmacokinetic-pharmacodynamic modeling of antibacterial drugs. Pharmacol Rev 2013;65:1053-90.

47. Brussee JM, Calvier EA, Krekels EH, et al. Children in clinical trials: towards evidence-based pediatric pharmacotherapy using pharmacokineticpharmacodynamic modeling. Expert Rev Clin Pharmacol 2016;9:1235-44.

48. Minto C, Schnider T. Expanding clinical applications of population pharmacodynamic modelling. Br J Clin Pharmacol 1998;46:321-33.

49. Sheiner LB, Steimer JL. Pharmacokinetic/pharmacodynamic modeling in drug development. Annu Rev Pharmacol Toxicol 2000;40:67-95.

50. Csajka C, Verotta D. Pharmacokinetic-pharmacodynamic modelling: history and perspectives. J Pharmacokinet Pharmacodyn 2006;33:227-79.

51. Wahlby U, Thomson AH, Milligan PA, Karlsson MO. Models for timevarying covariates in population pharmacokinetic-pharmacodynamic analysis. Br J Clin Pharmacol 2004;58:367-77.

52. Mager DE, Jusko WJ. Development of translational pharmacokineticpharmacodynamic models. Clin Pharmacol Ther 2008;83:909-12.

53. Barrett JS. Paediatric models in motion: requirements for model-based decision support at the bedside. Br J Clin Pharmacol 2015;79:85-96.

54. Vinks AA, Emoto C, Fukuda T. Modeling and simulation in pediatric drug therapy: application of pharmacometrics to define the right dose for children. Clin Pharmacol Ther 2015;98:298-308.

55. Ogungbenro K, Dokoumetzidis A, Aarons L. Application of optimal design methodologies in clinical pharmacology experiments. Pharm Stat 2009;8:239-52.

56. Earp JC, Mehrotra N, Peters KE, et al. Esomeprazole FDA approval in children with GERD: exposure-matching and exposure-response. J Pediatr Gastroenterol Nutr 2017;65:272-7.

57. Gamalo-Siebers M, Savic J, Basu C, et al. Statistical modeling for Bayesian extrapolation of adult clinical trial information in pediatric drug evaluation. Pharm Stat 2017;16:232-49.

58. Hattersley AT, Thorens B. Type 2 diabetes, SGLT2 inhibitors, and glucose secretion. N Engl J Med 2015;373:974-6.

59. Fossler MJ. Patient adherence: clinical pharmacology's embarrassing relative. J Clin Pharmacol 2015;55:365-7.

60. Matsui D. Current issues in pediatric medication adherence. Paediatr Drugs 2007;9:283-8.
61. Park LG, Howie-Esquivel J, Dracup K. Electronic measurement of medication adherence. West J Nurs Res 2015;37:28-49.

62. Tanna S, Lawson G. Dried blood spot analysis to assess medication adherence and to inform personalization of treatment. Bioanalysis 2014;6: 2825-38.

63. Hawwa AF, AlBawab A, Rooney M, Wedderburn LR, Beresford MW, McElnay JC. Methotrexate polyglutamates as a potential marker of adherence to long-term therapy in children with juvenile idiopathic arthritis and juvenile dermatomyositis: an observational, crosssectional study. Arthritis Res Ther 2015;17:295.

64. Gunning K, Rowan K. ABC of intensive care: outcome data and scoring systems. BMJ 1999;319:241-4.

65. Eberhart LH, Morin AM, Guber D, et al. Applicability of risk scores for postoperative nausea and vomiting in adults to paediatric patients. $\mathrm{Br} \mathrm{J}$ Anaesth 2004;93:386-92.

66. Wedderburn LR, Varsani H, Li CK, et al. International consensus on a proposed score system for muscle biopsy evaluation in patients with juvenile dermatomyositis: a tool for potential use in clinical trials. Arthritis Rheum 2007;57:1192-201.

67. Loeve M, Hop WC, de Bruijne M, et al. Chest computed tomography scores are predictive of survival in patients with cystic fibrosis awaiting lung transplantation. Am J Respir Crit Care Med 2012;185: 1096-103.

68. van Dijk M, de Boer JB, Koot HM, Tibboel D, Passchier J, Duivenvoorden HJ. The reliability and validity of the COMFORT scale as a postoperative pain instrument in 0 to 3 -year-old infants. Pain 2000;84:367-77.

69. Stevens B, Johnston C, Petryshen P, Taddio A. Premature infant pain profile: development and initial validation. Clin J Pain 1996;12:13-22.

70. Cohen J. Weighted kappa: nominal scale agreement with provision for scaled disagreement or partial credit. Psychol Bull 1968;70:213-20.

71. Ista E, de Hoog M, Tibboel D, van Dijk M. Implementation of standard sedation management in paediatric intensive care: effective and feasible? J Clin Nurs 2009;18:2511-20.

72. Ueckert S, Plan EL, Ito K, et al. Improved utilization of ADAS-cog assessment data through item response theory based pharmacometric modeling. Pharm Res 2014;31:2152-65.

73. Finnegan LP, Connaughton JF Jr., Kron RE, Emich JP. Neonatal abstinence syndrome: assessment and management. Addict Dis 1975;2:141-58.

74. Ista E, van Dijk M, Tibboel D, de Hoog M. Assessment of sedation levels in pediatric intensive care patients can be improved by using the COMFORT "behavior" scale. Pediatr Crit Care Med 2005;6:58-63.

75. Steffes M, Cleary P, Goldstein D, et al. Hemoglobin A1c measurements over nearly two decades: sustaining comparable values throughout the Diabetes Control and Complications Trial and the Epidemiology of Diabetes Interventions and Complications study. Clin Chem 2005;51: 753-8.

76. Daneman D. Type 1 diabetes. Lancet 2006;367:847-58.

77. Weykamp C, John WG, Mosca A. A review of the challenge in measuring hemoglobin A1c. J Diab Sci Technol 2009;3:439-45.

78. Aslan B, Gun-Munro J, Flynn GJ. Role of proficiency testing in monitoring of standardization of hemoglobin A1c methods. Accredit Qual Assur 2012;17:419-24.

79. Food and Drug Administration Guidance for Industry. Bioanalytical Method Validation, 2001 http://www.fda.gov/downloads/Drugs/.../Guidances/ ucm070107.pdf.

80. Dunne J, Murphy D, Wharton G. The globalization of pediatric trials: should we be worried? Pediatrics 2011;127:e252-3 author reply e3-4.

81. Savage WJ, Everett AD. Biomarkers in pediatrics: children as biomarker orphans. Proteomics Clin Appl 2010;4:915-21.

82. Karbasy K, Lin DC, Stoianov A, et al. Pediatric reference value distributions and covariate-stratified reference intervals for 29 endocrine and special chemistry biomarkers on the Beckman Coulter Immunoassay Systems: a CALIPER study of healthy community children. Clin Chem Lab Med 2015;54:643-57.

83. Sigdel TK, Gao X, Sarwal MM. Protein and peptide biomarkers in organ transplantation. Biomark Med 2012;6:259-71. 
84. Smits A, De Cock RF, Cossey V, Knibbe CA, Allegaert K. Is indirect hyperbilirubinemia a useful biomarker of reduced propofol clearance in neonates? Biomark Med 2012;6:283-9.

85. Buijs EA, Zwiers AJ, Ista E, Tibboel D, de Wildt SN. Biomarkers and clinical tools in critically ill children: are we heading toward tailored drug therapy? Biomark Med 2012;6:239-57.

86. Kwiatkowski DM, Goldstein SL, Krawczeski CD. Biomarkers of acute kidney injury in pediatric cardiac patients. Biomark Med 2012;6:273-82.

87. McWilliam SJ, Antoine DJ, Sabbisetti V, et al. Reference intervals for urinary renal injury biomarkers KIM-1 and NGAL in healthy children. Biomark Med 2014;8:1189-97.

88. McWilliam SJ, Antoine DJ, Sabbisetti V, et al. Mechanism-based urinary biomarkers to identify the potential for aminoglycoside-induced nephrotoxicity in premature neonates: a proof-of-concept study. PLoS ONE 2012;7:e43809.

89. Schiffl H, Lang SM. Urinary biomarkers and acute kidney injury in children: the long road to clinical application. Pediatr Nephrology 2013;28:837-42.

90. Coster WJ. Making the best match: selecting outcome measures for clinical trials and outcome studies. Am J Occup Ther 2013;67:162-70.

91. De Vet HC, Terwee CB, Mokkink LB, Knol DL. Measurement in Medicine: a Practical Guide. Cambridge University Press, 2011.
92. Sinha IP, Altman DG, Beresford MW, et al. Standard 5: selection, measurement, and reporting of outcomes in clinical trials in children. Pediatrics 2012;129 (Suppl 3): S146-52.

93. Williamson PR, Altman DG, Blazeby JM, et al. Developing core outcome sets for clinical trials: issues to consider. Trials 2012;13:132.

(i)

This work is licensed under a Creative Commons Attribution 4.0 International License. The images or other third party material in this article are included in the article's Creative Commons license, unless indicated otherwise in the credit line; if the material is not included under the Creative Commons license, users will need to obtain permission from the license holder to reproduce the material. To view a copy of this license, visit http://creativecommons.org/licenses/by/4.0/

(c) The Author(s) (2018) 